\title{
Impact of the mobile terminal scheme on millimeter- wave radio over fiber systems based on photonic heterodyning techniques
}

\author{
Ivan Aldaya $^{1,2} \cdot$ Gabriel Campuzano $^{3}$ - Carolina Del-Valle-Soto ${ }^{4}$ (D) \\ Alejandro Aragón-Zavala ${ }^{5}$ Gerardo Castañón ${ }^{3}$
}

Received: 27 January 2017/ Accepted: 17 May 2017/Published online: 26 May 2017

(C) Springer Science+Business Media New York 2017

\begin{abstract}
The use of communication networks relying on millimeter-wave (mm-wave) wireless links promises a great capacity enhancement as well as improved security. However, given the high-directivity of mm-wave links, coverage requirements are difficult to meet unless the network is assisted by an infrastructure. Given its low-cost, powerefficiency, and high capacity, radio over fiber has emerged as a strong candidate for the implementation of such infrastructure. Among the different generation techniques, photonic heterodyning has attracted considerable attention due to its capacity to generate radio frequency (RF) signals in the entire microwave/mm-wave range without requiring broadband electro-optical modulator. However, the RF signals generated using these techniques suffer from significant phase noise, a major impairment that degrades the system performance. In this paper we study two approaches to overcome this limitation: (1) the use of optical sideband injection locking (OSBIL) to generate tones with highly correlated phase noise and (2) heterodyning independent lasers in combination with a mobile terminal (MT) that is insensitive to the phase of the RF signal. A qualitative comparison between the two techniques in terms of MT sensitivity to the RF phase noise, the power sensitivity, and base station power efficiency leads to the conclusion that OSBIL is more
\end{abstract}

Ivan Aldaya

ivan.a.aldaya@ieee.org

Carolina Del-Valle-Soto

cvalle@up.edu.mx

1 Gleb Wataghin Physics Institute, University of Campinas, Campinas, SP, Brazil

2 São Paulo State University (UNESP), Campus of São João da Boa Vista, São João da Boa Vista, SP, Brazil

3 Department of Electrical and Computer Engineering, Tecnológico de Monterrey, Monterrey, NL, Mexico

4 Facultad de Ingeniería, Universidad Panamericana, Prolongación Calzada Circunvalación Poniente 49, 45010 Zapopan, Jalisco, Mexico

5 Department of Electrical and Computer Engineering, Tecnológico de Monterrey, Querétaro, Qro, Mexico 
suitable for networks with medium-range node separation, whereas heterodyning of independent laser with phase-insensitive MT is a cost-efficient solution for networks where nodes are closer to each other.

Keywords Radio over fiber · Millimeter-wave signals · Photonic heterodyning · Sideband injection locking

\section{Introduction}

Driven by the spectrum scarcity of the ultra-high frequency (UHF) band, millimeter-wave (mm-wave) communication systems have gained an increasing attention in the last years, as proven by several standards: WiMAX standard was published (Andrews et al. 2007) including an operation mode in the unlicensed $60 \mathrm{GHz}$ band for point-to-point fixed wireless link. In 2009, ECMA 387 (2010) and IEEE 802.15.3c (2009) standards for wireless personal area networks (WPAN) were released, both using the $60 \mathrm{GHz}$ band. The same year the WiGig trade association was constituted. The aim of WiGig was to develop a standard for high-capacity wireless local area networks (WLANs), upgrading the wellknown WiFi IEEE 802.11 standard to support future multi-Gbps applications, using also the $60 \mathrm{GHz}$ frequency band (Hansen 2011). Other bands are being explored for mobile broadband internet access, such as the frequency band around $38 \mathrm{GHz}$, which has been proposed for 5G cellular systems (Rappaport 2013). At mm-wave frequencies, both the free-space (Rappaport 2007; Saunders and Aragon-Zavala 2007) and atmospheric attenuation (Giannetti et al. 1999) are higher than at lower frequencies, reducing both cochannel interference and cell size. Even if cell reduction enhances the aggregated capacity in both WLANs and broadband mobile internet systems, the use of pico and femtocells is a double edge sword since the capacity is improved at the expense of a significant increase of the number of base stations (BSs). Consequently, in mm-wave WLANs and broadband mobile systems, BSs must be kept as simple and power efficient as possible. Radio over fiber (RoF) has emerged as a green and cost-efficient technology for the generation and distribution of mm-wave multi-Gbps signals to remote BSs (Hall et al. 2013). In RoF systems the modulation, radio frequency (RF) generation, and resource allocation tasks are concentrated at the central station (CS) and, therefore, BSs are extremely simple, they only convert the optical signal into the electrical domain, amplify it, and radiate it to the mobile terminals (MTs).

One of the most challenging tasks in RoF systems is the generation of an optical signal that, after photodetection, results in the desired mm-wave signal. A wide range of remote mm-wave generation techniques has been proposed in recent years (Berceli and Herczfeld 2010; Beas et al. 2013). However, most of them require tailored components (Wake et al. 1995; Khawaja and Cryan 2010) or expensive high-speed optical modulators (Jia et al. 2008; Ng'oma and Sauer 2009), which limit the maximum mm-wave frequency that can be achieved. An attractive alternative is the heterodyning of two lasers that are tuned to emit at frequencies that differ by the desired RF frequency (González-Insua et al. 2010; Timofeev et al. 1998). The main advantage of the heterodyne technique is its flexibility to tune the generated RF frequency over the entire microwave/mm-wave band by the use of electrical or thermal control, being limited by the photodetector (PD) bandwidth. However, due to laser frequency drift, the beating signal may present significant frequency variation that, given the relatively slow nature of the phenomenon, can be reduced by improving its 
temperature and electrical control (Logan 1997). Another drawback of the heterodyning technique is the phase noise in the photogenerated current. The RF phase noise increases with laser linewidth and, when down-converting to baseband with a heterodyning MT, the phase noise of the RF signal is converted to amplitude noise, degrading the received signal quality, which further impoverishes the link quality indicator (LQI) at the MT. As with any other impairment, the impact of the phase noise on system performance depends on the employed modulation format. Due to its robustness to multipath effect on the wireless link, its high spectral efficiency, and resource allocation flexibility (Hwang et al. 2009; Daniels et al. 2007), orthogonal frequency division multiplexing (OFDM) has been adopted in most recent communication standards, also at mm-wave frequencies (Hansen 2011). In OFDM systems, the carrier phase noise induces a random constellation rotation (common phase error) as well as intercarrier interference (Zou et al. 2007), deteriorating the signal quality. Pilot-assisted equalization, where some subcarriers are left unmodulated to equalize the channel (Shieh and Djordjevic 2010), can correct this common phase error by estimating the average-phase on each OFDM symbol (Zou et al. 2007). However, pilotassisted equalization is not capable of compensating the constellation dispersion caused by intercarrier interference (Garcia-Armada 2001). It is important noting that the power relation between common phase error and intercarrier interference strongly depends on the OFDM symbol period: a shorter OFDM symbol allows a more accurate time-resolved phase estimation, resulting in a lower intercarrier interference and higher robustness to the RF phase noise. Consequently, from a phase noise point of view, the transmission of OFDM signals with relatively low bit rates and low number of bits per symbol represents the worst-case scenario. Thereafter we will limit our study to OFDM signals at $640 \mathrm{Mbps}$ with quadrature phase shift keying (QPSK) modulation. In this context, there are three possible design approaches to compensate the effect of phase noise: (1) using of narrowlinewidth lasers, which is expensive and not easy to integrate; (2) forcing correlation between the two optical beating tones, e.g. by using optical sideband injection locking (OSBIL); or (3) using phase-insensitive MTs based either on self-heterodyning (SH) or envelope detection (ED), which relaxes laser linewidth requirements.

Our work reviews the last two approaches: OSBIL and the use of uncorrelated lasers in combination with a phase-insensitive MT, extending and comparing our previous work (Aldaya et al. 2014a, b) that considered each technique independently. In this way, the implications of choosing OSBIL or uncorrelated tones goes beyond the CS design, as it imposes a particular MT detection scheme. The paper is organized as follows: in Sect. 2, we develop expressions relating the RoF network requirements and the RF front-end architecture. This allows the identification of the best combinations of MT architecture and photonic RF generation technique, which are further analyzed in the following sections. In Sect. 3, an RoF system based on OSBIL is numerically analyzed in terms of its sensitivity to the fiber chromatic dispersion and wireless range. Afterwards, in Sect. 4, experimental results are presented demonstrating that ED based MTs allow heterodyning of independent lasers with a combined linewidth close to $1 \mathrm{MHz}$. Finally, Sect. 5 concludes the paper.

\section{RF generation using optical heterodyning techniques}

In this section, we start with the derivation of the power spectral density of the photocurrent generated when two tones are heterodyned in a PD (Gallion and Debarge 1984; Gliese et al. 1996). This expression includes a differential delay between the beating tones, 
allowing de-correlation studies and is valid for RF signal generation using either OSBIL or independent lasers. The effect of phase noise on MTs using ED or heterodyning downconversion is then analyzed. The Section concludes with a discussion of when it is more suitable to use OSBIL and when it is to use heterodyning of independent lasers.

\subsection{Phase noise of the photogenerated current}

Let us consider two lasers emitting at angular frequencies $\omega_{1}$ and $\omega_{2}$. Assuming that the lasers are biased well above their threshold currents, the emitted fields can be modeled as constant-amplitude quasi-monochromatic fields (Gallion and Debarge 1984). Hence, the total optical field, $E(t)$, can be expressed as:

$$
E(t)=E_{1} \exp j\left[\omega_{1} t+\phi_{1}(t)\right]+E_{2} \exp j\left[\omega_{2} t+\phi_{2}(t)\right]+c . c .,
$$

where $E_{1}$ and $E_{2}$ are the optical field amplitudes of the lasers emitting at $\omega_{1}$ and $\omega_{2}$, respectively, and c.c. denotes the complex conjugation of the precedent term. Considering stability of long-term frequency fluctuations, $\phi_{1}(t)$ and $\phi_{2}(t)$ account only for the phase noise. The phase noise of an optical source is generally given in terms of its linewidth, the full width at half maximum (FWHM), measured in the frequency domain.

The power spectral density of the emitted optical field can be obtained by a semiclassical analysis, giving as a result a Lorentzian profile (Buus 1990):

$$
S_{E_{1 \mid 2}}(\omega)=\frac{\Delta \omega^{2} E_{1 \mid 2}^{2}}{\Delta \omega^{2}+\left(\omega-\omega_{1 \mid 2}\right)^{2}},
$$

where $E_{1 \mid 2}$ is the amplitude of the field, $\omega_{1 \mid 2}$ is the emission frequency, and $\Delta \omega$ denotes the linewidth given by:

$$
\Delta \omega=\frac{R_{S P}}{4 \pi S}\left(1+\alpha^{2}\right) .
$$

Equation (3) shows the dependency of linewidth on design-related parameters, such as linewidth enhancement factor, $\alpha$, and the spontaneous emission rate, $R_{S P}$. It also shows the dependency of operational parameters, like the photon density $S$. For distributed feedback (DFB) lasers, typical linewidths are in the order of $\mathrm{MHz}$, whereas in external cavity lasers (ECL) they decrease down to hundreds of $\mathrm{kHz}$.

The expression for the power spectral density of the photogenerated current, $S_{I}(\omega)$, after heterodyning two optical tones with phase noise was developed in Gallion and Debarge (1984). In Gliese et al. (1996) the expression for $S_{I}(\omega)$ was slightly modified neglecting low frequency components that are not of interest, resulting in the expression of Eq. (4).

$$
\begin{aligned}
S_{I}= & R^{2} \cdot E_{0}^{4} \cdot \delta\left(\omega-\omega_{R F}\right) \cdot \exp (-2 \Delta \omega \Delta \tau)+\frac{4 \pi^{2} \Delta \omega R^{2} E_{0}^{4}}{\left[(2 \Delta \omega)^{2}+\left(\omega-\omega_{R F}\right)^{2}\right]} \\
& \times\left\{1-\exp (-2 \Delta \omega \Delta \tau)\left[\cos \left(\left(\omega-\omega_{R F}\right) \Delta \tau\right)+\frac{2 \Delta \omega \sin \left(\left(\omega-\omega_{\mathrm{RF}}\right) \Delta \tau\right)}{\omega-\omega_{R F}}\right]\right\}
\end{aligned}
$$

In Eq. (4), $\omega_{R F}$ stands for the frequency difference, $\omega_{2}-\omega_{1}$, and $\Delta \tau$ accounts for the degree of correlation between the optical tones: $\Delta \tau=0$ for perfect correlation, while $\Delta \tau=\infty$ results in complete de-correlation (Gliese et al. 1996). Equation (4) has two terms; the first one, a discrete component corresponding to the correlated part, and the second term is a modified Lorentzian component associated to the uncorrelated part. For 
perfect correlation then, the Lorentzian term disappears, resulting in a spectrally-pure tone. On the other hand, in the complete de-correlated case, the power spectrum acquires a Lorentzian shape with a linewidth equal to twice the linewidth of the tones, $2 \Delta \omega$ (for the general case of two tones with different linewidths, the linewidth of the photogenerated RF tone is $\Delta \omega_{1}+\Delta \omega_{2}$, where $\Delta \omega_{1}$ and $\Delta \omega_{2}$ are the linewidths of each tone).

In an OSBIL transmitter, the phase noise of the two beating tones is correlated, and therefore, highly spectrally-pure RF signals are generated, even with DFB lasers. Conversely, when beating lasers with uncorrelated phase noises, the phase of the RF signal depends on the laser linewidth and, consequently, RF phase noise can only be reduced by decreasing the phase noise of the lasers.

\subsection{Frequency drift of the photogenerated current}

Alongside with the phase noise, the photogenerated current may be subject to frequency fluctuation caused by electrical and thermal drift of the heterodyning lasers. Given the high sensitivity of the emission wavelength to the temperature in semiconductor lasers, i.e. tens of $\mathrm{GHz}$ per degree celsius, thermal drift is usually claimed as the main source of frequency drift (Liu et al. 2005). Additionally, in order to improve the operation lifetime of the laser diodes, temperature control scheme is typically designed to operate with a hysteresis cycle (Brillant 2008). The frequency drift thereby occurs in the time scale of the temperature control, which is several seconds or even slower. This means that in a symbol period, the RF frequency may deviates from its nominal value but it remains constant. The effect on the photogenerated current can be easily accounted by substituting $\omega_{R F}$ in Eq. (4) by $\left\langle\omega_{R F}\right\rangle$, which is the average value of the beating frequency within the symbol period. In the case of OSBIL, each laser is clamped to a sideband. The frequencies at which these sidebands appear depend on two mechanisms: on the one hand, frequency fluctuation of the oscillator used to modulate the master that leads to the variation of frequency separation. This issue, however, is expected to have a negligible effect since electrical oscillators are generally equipped with frequency stabilization circuitry. On the other hand, the frequency drift of the master laser results in the frequency shift of the whole master laser emission spectrum. Fortunately, since the frequencies of all the sidebands are shifted equally, the frequency separation between sidebands does not vary. Consequently, performance degradation due to frequency separation is envisaged to be important only when independent lasers are beat.

\subsection{Effect of the MT scheme on RF spectral requirements}

Since high-frequency electronics is expensive and power inefficient, the front end of the MT, where the incoming RF signal is down coverted either to baseband or to a low intermediate frequency, $\omega_{I F}$, is a critical stage (González-Insua et al. 2009). This downconversion can be performed heterodyning the detected RF signal with the output of a local oscillator (Marcu et al. 2009) or, alternatively, employing a MT scheme that uses a carrier transmitted by the BS together with the modulation sideband (González-Insua et al. 2010). In the case of $\mathrm{SH}$, the transmitted carrier is used as a local oscillator, whereas in ED, the carrier is employed to avoid zero-crossing distortion. In both cases, the carrier and modulation sidebands are affected by the same phase noise and, therefore, when heterodyning both, the effect of phase noise disappears.

In order to understand the main impairments affecting the system performance when different MT schemes are used, we first derive an expression for the signal that is down 
converted using heterodyning MT, and then, another expression for the down converted signal using an ED-based MT. For the rest of the subsection, it is useful to introduce the complex low-pass modulating signal, $m(t)$, which can be expressed in terms of its in-phase, $m_{I}(t)$, and quadrature, $m_{Q}(t)$, components:

$$
m(t)=m_{I}(t)+j \cdot m_{Q}(t) .
$$

Likewise, the passband modulating signal, $m^{\prime}(t)$, after upconverting $m(t)$ to $\omega_{I F}$ can be written as:

$$
m^{\prime}(t)=m_{I}(t) \cos \left(\omega_{I F} t\right)-m_{Q}(t) \sin \left(\omega_{I F} t\right),
$$

where the phase noise of the electrical oscillator has been neglected assuming that the major contribution to the total phase noise in the RF signal is generated in the up-conversion to mm-wave frequencies (Braun 1998).

\subsubsection{Down-conversion using a heterodyning $M T$}

When heterodyne detection at the MT is employed, suppressed carrier single sideband (SCSSB) modulation can be used for the radiated electrical signal. The main advantage of SCSSB in comparison to double sideband (DSB) is its higher spectral efficiency, whereas in comparison to single sideband with carrier (SSB+C), SC-SSB is more power efficient, since no power is wasted in transmitting the carrier. An SC-SSB signal can be expressed as:

$$
\begin{aligned}
s_{S C-S S B}(t)= & m_{I}(t) \cos \left(\omega_{R F}^{\prime} t+\Delta \phi(t)\right) \\
& -m_{Q}(t) \sin \left(\omega_{R F}^{\prime} t+\Delta \phi(t)\right)+n_{S S B}(t),
\end{aligned}
$$

where $\omega_{R F}^{\prime}=\omega_{R F}+\omega_{I F}$ is the central frequency of the modulation sideband and $\Delta \phi(t)$ represents the phase noise. $n_{S S B}(t)$ is an additive noise accounting for different noise sources such as the laser relative intensity noise, PD thermal and shot noises, and the noise induced by the electrical amplifier at the BS. For simplicity, we consider that the mm-wave signal is downconverted to $\omega_{I F}$ in a first stage:

$$
\begin{aligned}
s_{S C-S S B}^{\prime}(t)= & \sqrt{\frac{K}{L}} m_{I}(t) \cos \left(\omega_{I F} t+\Delta \phi(t)\right) \\
& -\sqrt{\frac{K}{L}} m_{Q}(t) \sin \left(\omega_{I F} t+\Delta \phi(t)\right)+n_{S S B}(t)
\end{aligned}
$$

where $K$ and $L$ are the mixing gain and path-loss, respectively, with $K / L>1$. In a second stage, the signal is further down converted from $\omega_{I F}$ to baseband where we get two components:

$$
s_{I}(t)=\sqrt{\frac{K}{L}} m_{I}(t) \cos \left(\Delta \phi^{\prime}(t)\right)+n_{I}(t)
$$

and

$$
s_{Q}(t)=\sqrt{\frac{K}{L}} m_{Q}(t) \cos \left(\Delta \phi^{\prime}(t)\right)+n_{Q}(t),
$$

$n_{I}(t)$ and $n_{Q}(t)$ are the in-phase and quadrature components of the equivalent noise added by the transmitter and receiver. $\Delta \phi^{\prime}(t)$ is the phase resulting from the sum of phase-noise 
(the RF signal and the local oscillator) and the frequency drift, which appears as a multiplicative noise term. Therefore, unless some phase correction mechanism is implemented, phase noise is the main impairment limiting reception performance. Indeed, even if pilotassisted equalization is implemented, for high levels of phase noise, equalization cannot accurately estimate the phase and signal quality is severely affected. On the other hand, when phase noise is small, the case of highly spectrally-pure signals with frequency stabilization, $\cos \left(\Delta \phi^{\prime}(t)\right) \approx 1$, and performance is then limited by receiver noise. The signal-to-noise ratios (SNRs) of the in-phase and quadrature signals are given by:

$$
S N R_{I}=\frac{K\left\langle m_{I}^{2}(t)\right\rangle}{L\left\langle n_{I}^{2}(t)\right\rangle} \text { and } S N R_{Q}=\frac{K\left\langle m_{Q}^{2}(t)\right\rangle}{L\left\langle n_{Q}^{2}(t)\right\rangle}
$$

with $\langle\cdot\rangle$ representing time average operation. Equation (10) makes clear the importance of the mixing gain in overcoming the effects of path loss on the received SNR and, consequently, in the wireless range.

\subsubsection{Down-conversion employing ED-based MT}

In contrast to heterodyning receivers, MTs based on ED require a carrier to be radiated alongside the modulation sideband. Consequently, Eq. (8) has to be modified to include the carrier:

$$
\begin{aligned}
s_{S S B+C}(t)= & A_{c} \cos \left(\omega_{R F} t+\Delta \phi(t)\right) \\
& +m_{I}(t) \cos \left(\omega_{R F}^{\prime} t+\Delta \phi(t)\right) \\
& -m_{Q}(t) \sin \left(\omega_{R F}^{\prime} t+\Delta \phi(t)\right)+n_{S S B}(t),
\end{aligned}
$$

where $A_{c}$ is the amplitude of the carrier satisfying $A_{c}>|m(t)|$ for any time. Mathematically, the output of the ED, $s_{E D}(t)$, can be calculated as the amplitude of the complex envelope of the SSB $+\mathrm{C}$ signal, $\tilde{s}_{S S B+C}(t)$, and considering a loss term, $L_{E D}$, and additive noise, $n_{E D}(t)$ :

$$
s_{E D}(t)=\frac{\sqrt{\left(\operatorname{Re}\left\{\tilde{s}_{S S B+C}(t)\right\}\right)^{2}+\left(\operatorname{Im}\left\{\tilde{s}_{S S B+C}(t)\right\}\right)^{2}}}{\sqrt{L_{P L} L_{E D}}}+n_{E D},
$$

with $\tilde{s}_{S S B+C}(t)=s_{S S B+C}(t)+j H T\left\{s_{S S B+C}(t)\right\} . s_{E D}(t)$ can be written in a more compact way as:

$$
s_{E D}(t)=\frac{\sqrt{\left(s_{S S B+C}(t)\right)^{2}+\left(H T\left\{s_{S S B+C}(t)\right\}\right)^{2}}}{\sqrt{L_{P L} L_{E D}}}+n_{E D} .
$$

Applying the Bedrosian theorem for high-low pass signals (Bedrosian 1962) and considering that $\left\langle n_{S S B}(t)^{2}\right\rangle \ll\left\langle|m(t)|^{2}\right\rangle$ and that $\left\langle n_{E D}(t)^{2}\right\rangle \ll A_{c}^{2}$, $s_{S S B+C}(t)$ is approximated by:

$$
s_{E D}(t) \approx \frac{A_{c}+m^{\prime}(t)}{\sqrt{L_{P L} L_{E D}}}+n_{E D}(t) .
$$

The output of the ED appears at $\omega_{I F}$ so a second frequency down-conversion must be performed: 


$$
s_{I}(t) \approx \frac{m_{I}(t)}{\sqrt{L_{P L} L_{E D}}}+n_{I}(t) \text { and } s_{Q}(t) \approx \frac{m_{Q}(t)}{\sqrt{L_{P L} L_{E D}}}+n_{Q}(t),
$$

where $n_{I}(t)$ and $n_{Q}(t)$ are the in-phase and quadrature components of $n_{E D}(t)$, respectively. From the previous expressions, it is clear that the downconverted baseband signals are not affected by the phase noise of the RF signal and, therefore, RF signals with relatively high phase noise can be down-converted using an ED-based MT. Following the same reasoning, we could expect that frequency drift neither affects the system performance. Nevertheless, if the bandwidth limitation of the RF signal is considered, an excessive frequency drift would cause part of the signal to be filtered out and, thus, the system performance is compromised. Unfortunately, even if ED-based downconversion is not affected by phase noise, it is severely affected by the $L_{E D}$ and $n_{E D}(t)$ as can be seen in the expression for $S N R_{I}$ and $S N R_{Q}$ :

$$
S N R_{I}=\frac{\left\langle m_{I}(t)^{2}\right\rangle}{L_{P L} L_{E D}\left\langle n_{I}(t)^{2}\right\rangle} \quad \text { and } \quad S N R_{Q}=\frac{\left\langle m_{Q}(t)^{2}\right\rangle}{L_{P L} L_{E D}\left\langle n_{Q}(t)^{2}\right\rangle} .
$$

Comparing the SNR in Eqs. (10) with (16), it is evident that ED-based receiver is more severely affected by the wireless path losses and the receiver noise, which results in a poorer wireless range.

\subsection{OSBIL versus heterodyning of uncorrelated tones}

Table 1 summarizes the pros and cons of the two heterodyning techniques as well as the MT schemes. In both cases, there is a tradeoff between cost and performance: OSBIL outperforms heterodyning of independent lasers in terms of spectral purity but it is more difficult to integrate and requires a higher number of optical components, which increases its cost. Regarding MT schemes, heterodyning MTs have better power sensitivity than EDbased MTs. However, the former requires spectrally pure RF signals and is more expensive.

\begin{tabular}{|c|c|c|c|c|c|}
\hline & \multicolumn{2}{|c|}{ OSBIL } & $\begin{array}{l}\text { Indep. } \\
\text { lasers }\end{array}$ & \multicolumn{2}{|l|}{ Implications } \\
\hline $\begin{array}{l}\text { Spectral } \\
\text { purity }\end{array}$ & \multicolumn{2}{|c|}{ Good } & Poor & \multicolumn{2}{|c|}{ When using independent lasers it depends on the laser linewidth } \\
\hline Integrability & \multicolumn{2}{|c|}{ Difficult } & Easy & \multicolumn{2}{|c|}{ OSBIL is difficult to integrate because of the required isolator } \\
\hline \multirow[t]{2}{*}{ Cost } & High & & Low & OSBIL requires & additional laser \\
\hline & \multicolumn{3}{|c|}{ Heterodyning MT } & ED-based MT & Implications \\
\hline \multicolumn{2}{|l|}{ RF phase noise } & \multicolumn{2}{|c|}{ Sensitive } & Insensitive & $\begin{array}{l}\text { ED-based MTs are required for RF signals } \\
\text { with high phase noise }\end{array}$ \\
\hline \multicolumn{2}{|c|}{ Carrier transmission } & \multicolumn{2}{|c|}{ Not required } & Required & $\begin{array}{l}\text { Better energy efficiency of heterodyning MT } \\
\text { since not power is wasted in the carrier }\end{array}$ \\
\hline \multicolumn{2}{|l|}{ Power sensitivity } & \multicolumn{2}{|c|}{ Good } & Poor & $\begin{array}{l}\text { Heterodyning MT allows higher path losses } \\
\text { and, consequently, longer wireless links }\end{array}$ \\
\hline Cost & \multicolumn{3}{|c|}{ High } & Low & $\begin{array}{l}\text { ED-based MTs are more suitable for low } \\
\text { cost applications }\end{array}$ \\
\hline
\end{tabular}

Table 1 Comparison between heterodyning and ED-based MTs 
There are four possible combinations of generation techniques with MT schemes: (1) heterodyning of independent lasers with a heterodyning MT is not a feasible combination since the high phase noise of the generated RF signal would degrade system performance. (2) OSBIL with ED-based MT is a combination that works, but it is not logical because the cost of the OSBIL transmitter is not justified. (3) OSBIL with a heterodyning MT is a logical approach and, therefore, is analyzed in Sect. 3. The OSBIL cost is justified because a spectrally-pure RF signal allows the use of a highly sensitive MT. The expensive implementation is compensated by its high performance that a priori would allow midrange wireless applications, including mobile broadband internet access. (4) The last combination corresponds to heterodyning independent lasers with an ED-based MT. This approach, studied in Sect. 4, is interesting because the phase insensitivity of ED enables the use of non-narrow linewidth lasers, which reduces system cost. The main drawbacks are the power inefficiency in the BS (a large proportion of the RF power is assigned to the carrier) and the low sensitivity of the MT. In consequence, this approach has the potential to support low-cost short-range wireless applications, such as WPAN and WLAN.

\section{Optical sideband injection locking}

OSBIL was first proposed in Goldberg et al. (1983) to generate RF tones with a sub-hertz linewidth at $10 \mathrm{GHz}$ and, subsequently in Braun (1998), the error-free transmission of a $155-$ Mbps offset QPSK signal in the $60 \mathrm{GHz}$ band was demonstrated. More recently, in Bhattacharya et al. (2002), the generation of a spectrally pure signal was analytically studied. In Fig. 1, an OSBIL transmitter is presented. A master laser (ML) is directly modulated by a microwave tone at $f_{i}$, a subharmonic of the desired mm-wave frequency, $f_{m m}$. The associated frequency modulation induces multiple modulation sidebands that appear at frequencies spaced $f_{i}$, as sketched in Fig. 1a. The feedback into the ML is avoided by means of an optical isolator. The output of the isolator is split employing a 50/ 50 optical splitter (SP) whose outputs are attenuated using independent variable optical attenuators (VOAs) to get the optimum power injection ratio, defined as the ratio of the output power of the slave laser operating in free-running to the injected power. The polarization matching between the ML and the slave lasers (SLs) is achieved by two polarization controllers (PC). One of the SLs is directly modulated with a passband signal centered at an intermediate frequency $f_{I F}$, whose electrical spectrum is shown in Fig. 1b. The second laser is left unmodulated to be used as reference. Figure $1 \mathrm{c}, \mathrm{d}$ show the spectrum of modulated and reference lasers, respectively. In order to overcome the fiberinduced differential delay between reference and modulated signals, a delay line (DL) is included in one of the arms. The laser bias currents and temperatures are set to control the frequency detuning, that is, the frequency difference between the free-running slave laser and the frequency of the injected light, locking $\mathrm{SL}_{1}$ and $\mathrm{SL}_{2}$ to modulation sidebands at $f_{0}$ and $f_{r e f}$, whose frequency difference corresponds to $f_{m m}$. The outputs of both lasers are combined, Fig. 1d, and sent over the optical distribution network to the BS.

\subsection{Simulation setup}

The system was studied using VPI Transmission Maker/Matlab co-simulation: the optical components such as lasers and optical fiber were simulated using VPI Transmission Maker, whereas OFDM modulation and demodulation were accomplished in Matlab. Figure 2a 


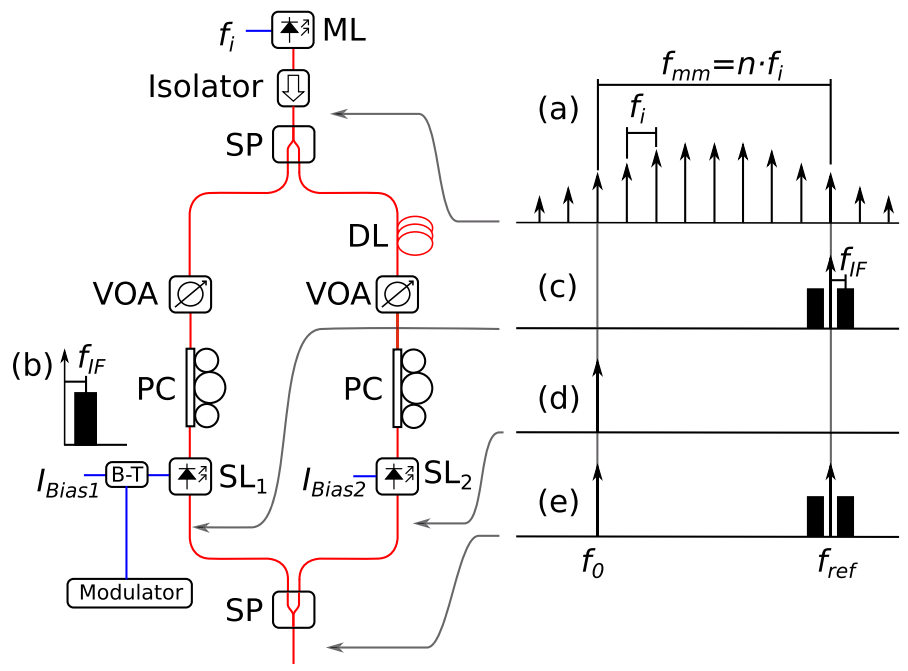

Fig. 1 OSBIL transmitter. $M L$ master laser, $S P$ optical splitter, $D L$ delay line, $V O A$ variable optical attenuator, $P C$ polarization controller, $S L_{1}$ modulated slave laser, $S L_{2}$ reference slave laser, and $B-T$ biastee. $f_{o}$ locking frequency of the modulated slave laser, $f_{\text {ref }}$ locking frequency of the reference slave laser, $f_{i}$ subharmonic frequency of the desired mmWave frequency $f_{m m}$, and $f_{I F}$ intermediate frequency. $I_{\text {Bias } 1 \text { and }}$ $I_{\text {Bias } 2}$ bias current of the modulated and slave laser, respectively. Schematic spectra at different points of the transmitter: $a$ spectrum at the output of the modulated master laser, $b$ electrical spectrum of the modulating signal, $c$ spectrum at the output of the modulated slave laser, $d$ spectrum at the output of the reference laser, and $e$ spectrum at the output of the OSBIL transmitter
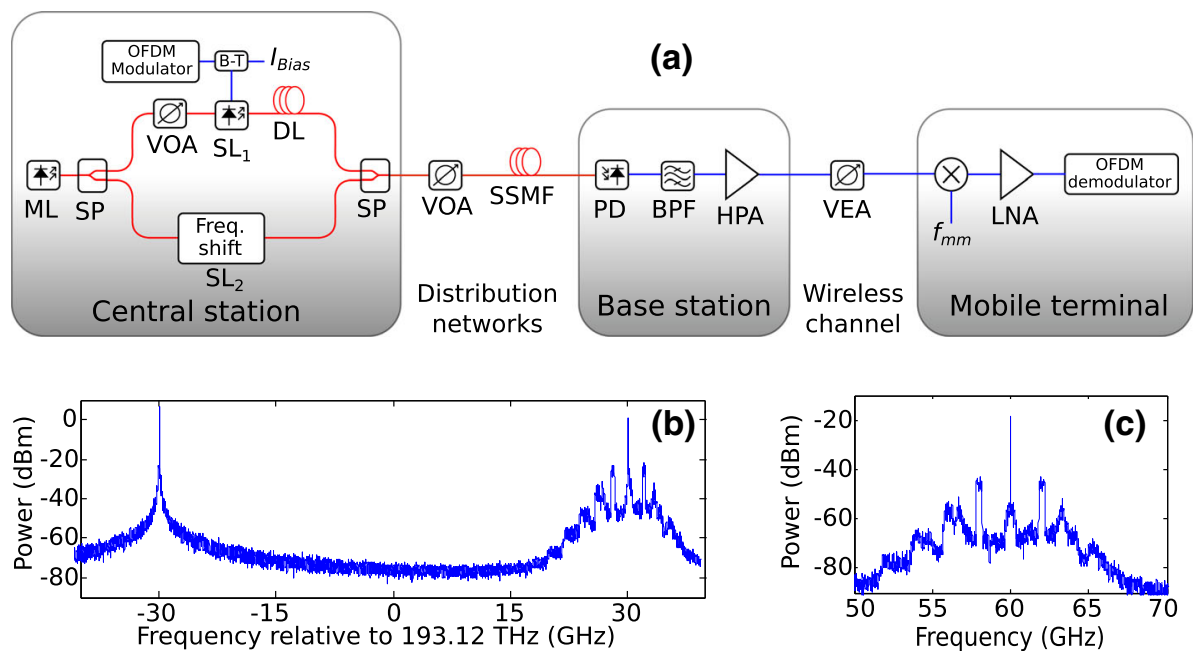

Fig. 2 a Simulation setup for an OFDM RoF system based on OSBIL. $M L$ master laser, SP optical splitter, $D L$ delay line, $V O A$ variable optical attenuator, $P C$ polarization controller, $S L_{1}$ modulated slave laser, $S L_{2}$ reference slave laser, and $B-T$ bias-tee, $S S M F$ standard single mode fiber, $P D$ photodetector, $B P F$ band-pass filter, $H P A$ high-power amplifier, $V E A$ variable electrical attenuator, and $L N A$ low-noise amplifier. $f_{m m}$ represents the desired mm-wave frequency. b Optical spectrum after the signal combination and $\mathbf{c}$ electrical spectrum of the photogenerated current 
shows the simulation setup for the analysis of an RoF system employing OSBIL. Figure $2 b$ represents the optical power spectrum after combination of both lasers, while Fig. 2c shows the spectrum after photodetection. The setup for the transmitter is based on that presented in Aldaya et al. (2014a), where the ML was modeled semianalytically, assuming a linewidth of $2 \mathrm{MHz}$. The output of the reference laser, SL1, was obtained shifting the frequency of the ML. The modulated slave laser, SL2, was modeled using the transmission line laser model (TLLM) with the parameters listed in Table 2. TLLM accounts for the longitudinal distribution of the optical power and carrier density, accurately modeling DFB lasers with $\lambda / 4$-shifted Bragg grating (Lowery 1989; Ghafouri-Shiraz 2003). The generated RoF signal was transmitted over an standard single mode fiber (SSMF) span and detected using a high bandwidth PD. The PD was followed by a band pass filter (BPF) centered at $62 \mathrm{GHz}$ with a bandwidth of $1 \mathrm{GHz}$, and a high-power amplifier (HPA) with a $30 \mathrm{~dB}$ gain. An electrical attenuator simulated the link budget accounting for transmitter and receiver antennas, as well as the path losses. Antenna's gain were set to $30 \mathrm{dBi}$ while the path loss was calculated according to expression (Saunders and Aragon-Zavala 2007):

$$
L_{P L}=\left(\frac{4 \pi \cdot d \cdot f_{m m}}{c}\right)^{2},
$$

with $d$ representing the length of the wireless link in $\mathrm{m}$, and $c$ the speed of light. The signal was downconverted to $f_{I F}$ heterodyning the incoming signal with a local electrical oscillator and removing the high-frequency components with a low-pass filter (LPF), before being demodulated in Matlab.

Table 2 Laser parameters used in TLLM simulation

\begin{tabular}{ll}
\hline & Value \\
\hline Physical parameters & \\
Cavity length $(L)$ & $316 \mu \mathrm{m}$ \\
Active region width $(W)$ & $2.5 \mu \mathrm{m}$ \\
Active region thickness $(T)$ & $210 \mathrm{~nm}$ \\
Nominal wavelength at $I_{t h}\left(\lambda_{0}\right)$ & $1550 \mathrm{~nm}$ \\
Facet reflectivities & $10^{-12}$ \\
Grating coupling $(\kappa)$ & $6000 \mathrm{~m}^{-1}$ \\
Linewidth enhancement factor $(\alpha)$ & 3 \\
Transparency carrier density $\left(N_{T r}\right)$ & $1.5 \times 10^{21} \mathrm{~m}^{-3}$ \\
Linear gain coefficient $(\epsilon)$ & $10^{-23} \mathrm{~m}^{3}$ \\
Internal loss $\left(\alpha_{\text {losses }}\right)$ & 3 \\
Monomolecular recombination $(A)$ & 0 \\
Bimolecular recombination $(B)$ & $10^{-16} \mathrm{~m}^{-3}$ \\
Auger recombination $(C)$ & $1.3 \times 10^{-41} \mathrm{~m}^{-6}$ \\
Reference refractive index $\left(n_{g}\right)$ & 3.7 \\
Simulation parameters & \\
Number of segments & 10 \\
Number of iterations & $2^{21}$ \\
Time step $(\Delta T)$ & $0.39063 \mathrm{ps}^{2}$ \\
Space step $(\Delta L)$ & $31.672 \mu \mathrm{m}$ \\
\hline
\end{tabular}




\subsection{Locking assessment and phase of the slave lasers}

Before generating a modulated signal, the proper locking of the SLs must be assessed, which can be done either in the frequency or time domain. We first look at the electrical spectrum obtained at the output of the PD. Figure 3a shows the spectra of the signals when the beating tomes are uncorrelated and when they are correlated by means of OSBIL (for the sake of clarity, two unmodulated tones were beat). As can be seen, the latter presents a much narrower linewidth, which in turns means that the generated signal has a significantly lower phase-noise than when two uncorrelated are used. The correlation between the two beating tones can be clearly appreciated in Fig. 3b where the time-resolved phases of the $\mathrm{ML}$ and the two SLs (one unmodulated used as a reference and another one modulated with a pure tone at $2 \mathrm{GHz}$ ) are shown. First of all, we should note the high degree of correlation between the ML and the unmodulated SL. However, the correlation is not perfect due to the presence of high frequency phase noise that is present outside the locking range. The comparison between the phase of the ML and the modulated SL is slightly more complicated because, even if optical locking reduces the modulation chirp, it cannot completely suppress it (Okajima et al. 2003). Therefore, a sinusoidal signal at the modulation frequency, i.e. $2 \mathrm{GHz}$, is superimposed on top of the phase noise of the SL. Fortunately, given the time scale difference of the laser phase noise and the modulation signal, both terms can be isolated. Therefore, we can see that the phase noise of the modulated SL is also highly correlated with that of the SL and, consequently, with that of the reference SL, showing that both SLs are locked.

\subsection{Optimization of injection conditions}

The power injection ratio $(R)$ and frequency detuning $(\Delta f)$ of the modulated laser were set based on the analysis carried out in Aldaya et al. (2014a), where injection conditions where optimized in terms of noise and nonlinear distortion considering the sideband asymmetry. Figure 4 summarizes the results for $f_{I F}=2 \mathrm{GHz}$ : Fig. $4 \mathrm{a}$, b show the SNR computed over a $1 \mathrm{~Hz}$ bandwidth in terms of the injection conditions for the upper and lower modulation sidebands, respectively. Three optimal regions where SNR is high can be identified: (1) lower modulation sideband with frequency detuning lower than $0 \mathrm{GHz}$ and power injection ratio between -34 and $-30 \mathrm{~dB}$, (2) upper modulation sideband with frequency detuning
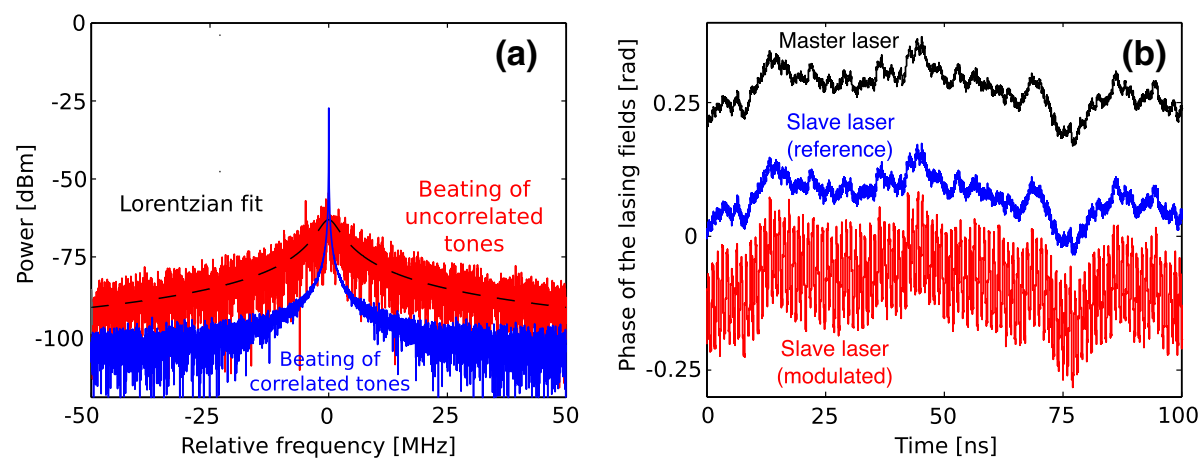

Fig. 3 a Spectra of the beating of two tones with uncorrelated and correlated phases. b Phase evolution of the master laser and slave lasers under no modulation (reference) and modulated with a pure sinusoidal tone (signal) 

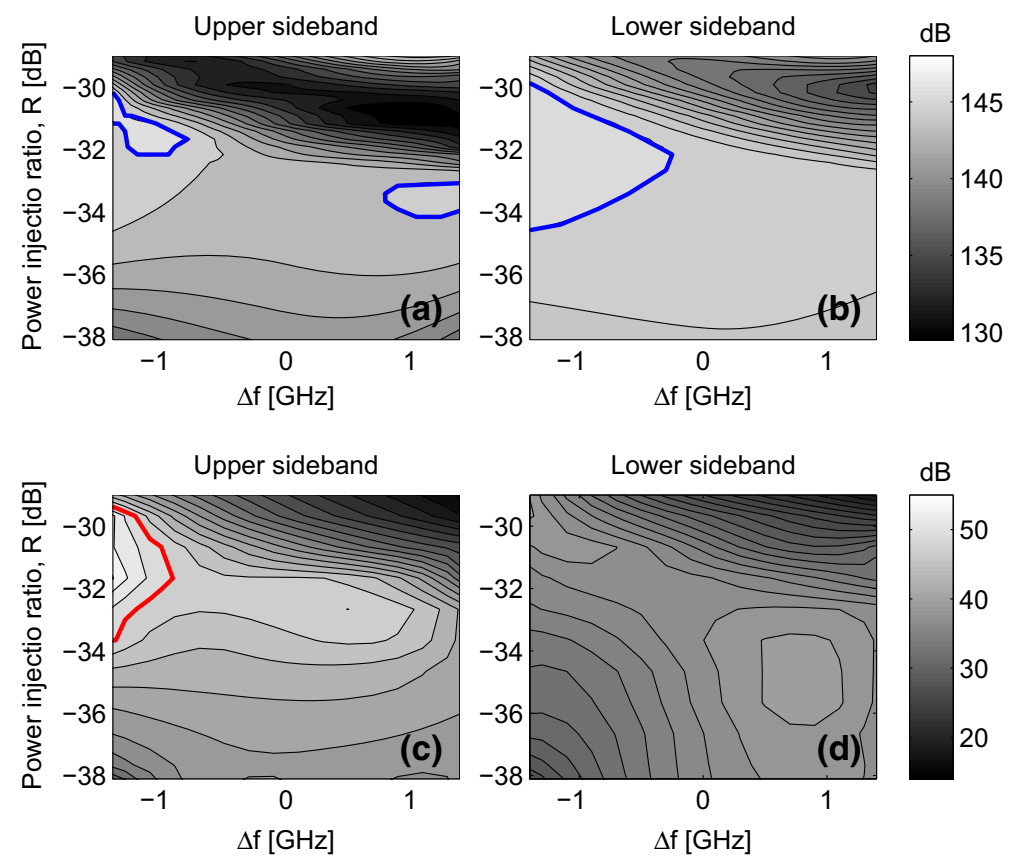

Fig. 4 Impact of modulation impairments in terms of injection conditions for upper and lower modulation sidebands: a, b SNR per Hz and c, $\mathbf{d}$ spurious-free dynamic range. Blue and red curves indicate regions of the parameter space where SNR is high and nonlinear distortion is low, respectively. (Color figure online)

$<-0.75 \mathrm{GHz}$ and power injection ratio ranging from -32 and $-31 \mathrm{~dB}$, and (3) upper modulation sideband with frequency detuning $>0.75 \mathrm{GHz}$ and power injection ratio between -34 and $-33 \mathrm{~dB}$. Figure $4 \mathrm{c}$, d represent the spurious-free dynamic range for 1 $\mathrm{MHz}$ bandwidth. From a nonlinear distortion point of view, the optimum injection conditions are frequency detuning $<-1 \mathrm{GHz}$ and a power injection ratio between -33 and $-29 \mathrm{~dB}$. Overlapping of optimal regions for the two considered impairments leads to optimal injection conditions: $\Delta f=1.2 \mathrm{GHz}$ and $R=-31 \mathrm{~dB}$. In addition, upper modulation sideband presents better performance.

The modulated laser was fed with a 640-Mbps OFDM signal having 128 subcarriers, 110 of which were modulated using QPSK modulation, while 18 subcarriers were left unmodulated to be used in channel transmittance estimation and phase noise correction through pilot-assisted equalization. Additionally, a cyclic prefix (CP) of $25 \%$ was included. The signal quality was assessed based on the error vector magnitude (EVM) of the received constellation, which is directly related to the bit error rate (BER) (Shafik et al. 2006). A $32 \%$ EVM was taken as threshold that, considering QPSK, corresponds to a BER of $10^{-3}$, commonly accepted as threshold limit in systems with forward error correction (FEC) coding.

\subsection{Generation of OFDM signals using the OSBIL technique}

Once the optimal injection conditions are set, the power of the signal used to modulate the laser, referred as driving power, is optimized. Figure 5 shows the EVM in terms of the driving power of the injected SL, considering no fiber and a 2.1-m wireless link. As 
Fig. 5 EVM versus laser driving power considering no fiber and a wireless link of $2.1 \mathrm{~m}$

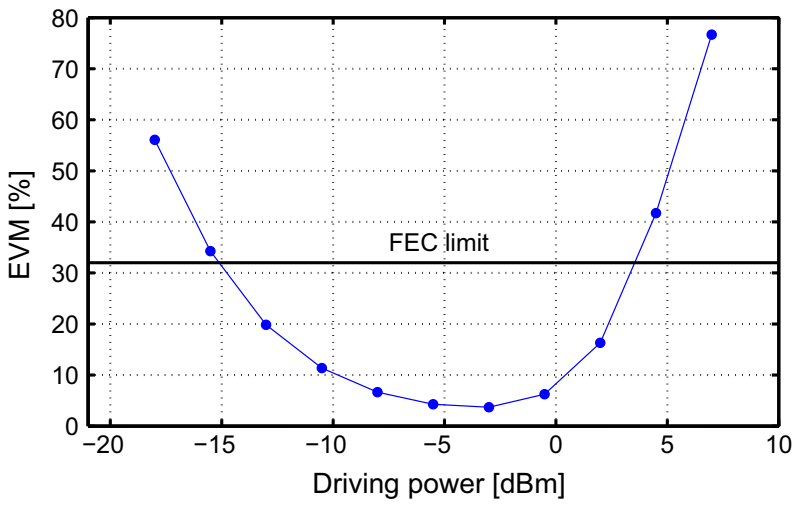

expected, at low driving power levels, a poor SNR results in high EVM. As the driving power was increased, the EVM decreased until a driving power of $-3 \mathrm{dBm}$ when nonlinear distortion became dominant. For higher driving power, the EVM increases rapidly due to the high peak to average power ratio (PAPR) of OFDM signals.

After optimizing the driving power, the effect of the fiber on the transmitted signal was analyzed using SSMF spans of different lengths (10, 26, and $37 \mathrm{~km})$ and varying the launched optical power. Simulation results, shown in Fig. 6, reveal that when the differential delay is not compensated by including a DL in one of the OSBIL transmitter arms, the power penalty at FEC limit induced by the partial decorration of the reference and modulated tones is around $1.5 \mathrm{~dB}$. However, when the DL was included, the power penalty decreases down to $0.3 \mathrm{~dB}$. Consequently, the performance degradation when no DL was included can be attributed mainly to the phase noise of the generated RF signal, since the robustness of OFDM signals to the chromatic dispersion is clear when DL was considered. The effect of the wireless distance on the received signal quality is shown in Fig. 7, for different fiber lengths. Since the output power of the HPA was kept constant, for longer fiber links, the radiated power decreases, and consequently, the maximum wireless range is shorter, $100 \mathrm{~m}$ for $10-\mathrm{km}$ SSMF, $45 \mathrm{~m}$ for $26-\mathrm{km}$, and $30 \mathrm{~m}$ for $37-\mathrm{km}$ fiber span.

Fig. 6 EVM versus received optical power for a $2.1-\mathrm{m}$ wireless link and different fiber lengths with and without including a delay line (DL) for differential delay compensation. Dashed lines indicate results with DL, while solid lines are for results without DL

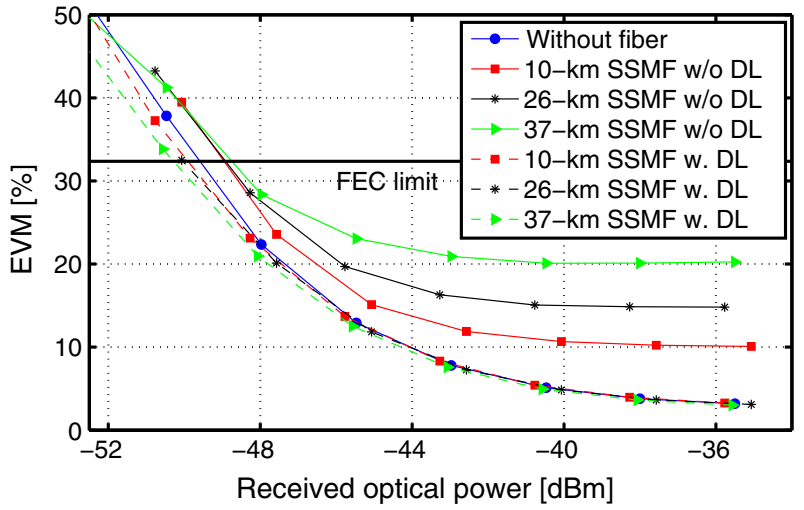




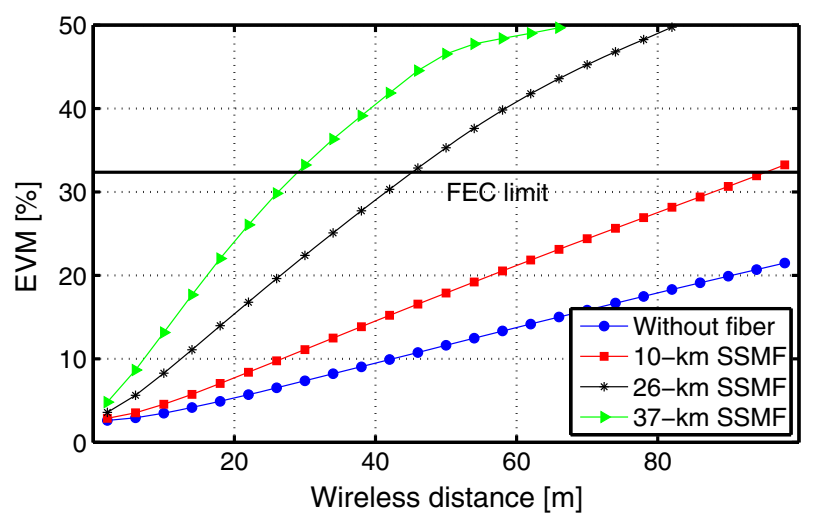

Fig. 7 EVM versus wireless distance for different fiber lengths

\section{Heterodyning of independent lasers}

An alternative to OSBIL is the heterodyning of lasers with uncorrelated phase noises. As concluded in Sect. 2, the effect of the phase noise of the RF signal can be overcome by employing MTs that are insensitive to the phase noise, i.e. ED-based MT. In this section we experimentally demonstrate the plausibility of heterodyning lasers with a combined linewidth close to $1 \mathrm{MHz}$ when an ED-based MT.

\subsection{Experimental setup}

The experimental setup is shown in Fig. 8. The OFDM signals were generated offline in Matlab using the same parameters as in Sect. 3 (110 data subcarriers, 18 pilots, QPSK subcarrier modulation, and a bit rate of $640 \mathrm{Mbps}$ ) and then loaded into a Tektronix AWG7122B Arbitrary Waveform Generator (AWG) operating at a sampling rate of 10.8 GS/s. The output of a continuous wave ECL, $L D_{1}$ in Fig. 8 was modulated using a $10-\mathrm{GHz}$ bandwidth dual-electrode Mach-Zehnder modulator (DE-MZM) driven by the in-phase and quadrature components of the OFDM signal and mixed in a 50/50 optical SP with another tunable ECL, $L D_{2}$ in Fig. 8. $L D_{1}$ and $L D_{2}$ were configured to emit at 1550 and 1549.51, respectively, resulting in a frequency separation of $60 \mathrm{GHz}$. Regarding the linewidth of the

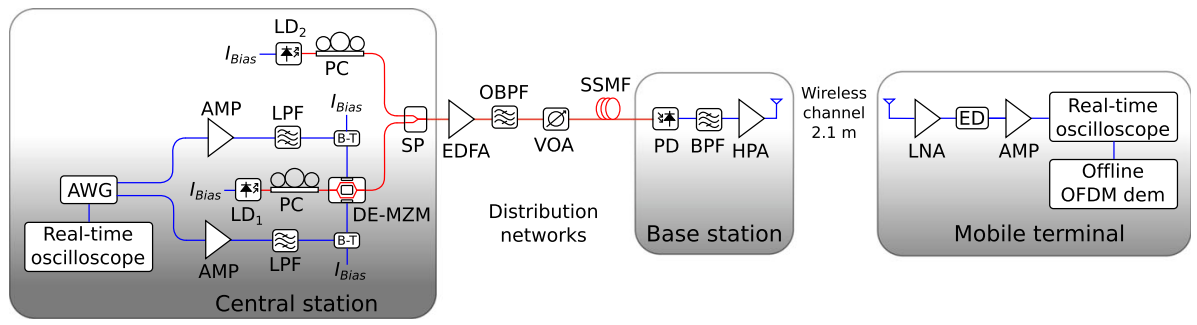

Fig. 8 Experimental setup. $A W G$ arbitrary waveform generator, $L D_{1}$ modulated laser diode, $L D_{2}$ reference laser diode, $S P$ optical splitter, $P C$ polarization controller, $E D F A$ erbium doped fiber amplifier, $O B P F$ optical bandpass filter, $V O A$ variable optical amplifier, $L P F$ low-pass filter, $B T$ bias-tee, $D E$ - $M Z M$ dualelectrode Mach-Zehnder modulator, SSMF standard single mode fiber, HPA high-power amplifier, LNA lownoise amplifier, $E D$ envelope detector, and $A M P$ electrical amplifier 
ECLs, $L D_{1}$ had a nominal wavelength at $10 \mathrm{dBm}$ of $100 \mathrm{kHz}$, whilst the linewidth of $L D_{2}$ was $800 \mathrm{kHz}$. The output of the combiner, was amplified using an Erbium doped fiber amplifier (EDFA), which was followed by an optical band-pass filter (OBPF) with a 0.8 $\mathrm{nm}$ bandwidth to remove out-of-band noise. The amplified signal was attenuated using a variable optical attenuator and transmitted over a span of SSMF to a 70-GHz bandwidth PD. The photogenerated current, $i_{P D}(t)$, was amplified using a 30-dB gain HPA and radiated by a $30-\mathrm{dBi}$ horn antenna. After a $2.1-\mathrm{m}$ wireless link, the signal was received by another 30-dBi horn antenna, whose output was amplified by an LNA before being downconverted to $f_{I F}$ using an ED. The downconverted signal was captured using a Tektronix DPO72004, 20-GHz real-time oscilloscope and demodulated in Matlab.

\subsection{Generation of OFDM signals using lasers with uncorrelated phase noise}

Figure 9 shows the EVM of the demodulated OFDM signal in terms of the driving power for a fixed launched optical power of $1 \mathrm{dBm}$, as well as the received constellations for (a) $-19.7 \mathrm{dBm}$, (b) $-9.2 \mathrm{dBm}$, and (c) $-6 \mathrm{dBm}$ driving powers. For low driving power the signals are severely affected by noise, resulting in bad performance. On the other hand, at high driving power, the nonlinear distortion induced by the MZM and postdetection electronics is dominant, affecting significantly the signal quality. As occurred in OSBIL transmitter, nonlinear distortion is particularly harmful in OFDM signals given their high PAPR, which results in a fast signal degradation.

The OFDM signal was then transmitted over an SSMF span and a 2.1-m wireless link while the launched optical power was swept by controlling the VOA. Figure 10 shows the obtained EVM curves for back-to-back, 10, 26, and 37-km fiber links. The perfect match between the different curves reveals that fiber dispersion does not affect signal quality, which can be attributed to the pilot assisted equalization and the inclusion of the CP. In regards to the nonlinear distortion, the Kerr effect of the fiber can be neglected since the dominant nonlinearities associated to the DE-MZM are dominant.
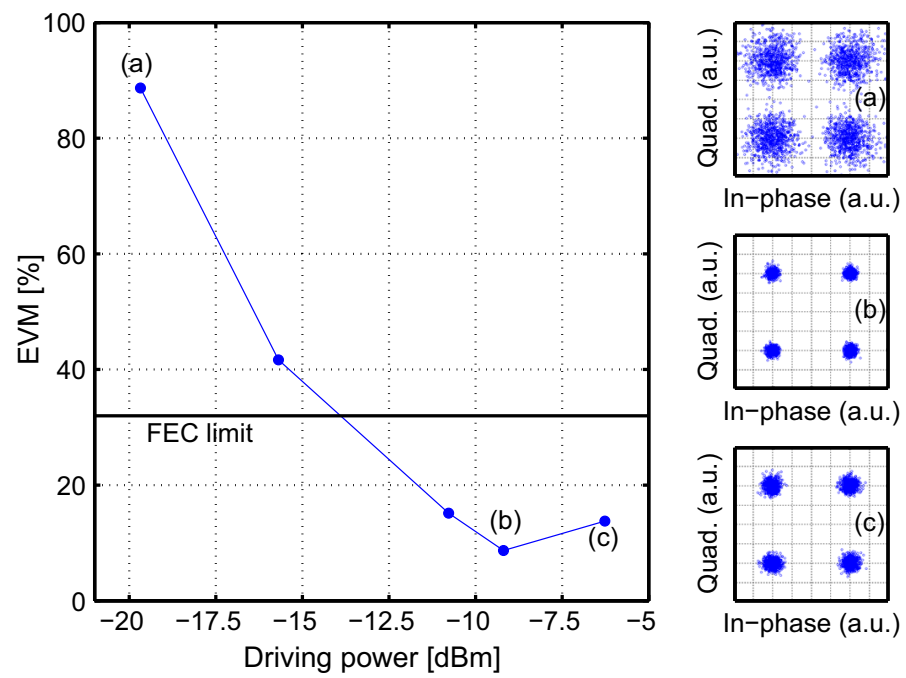

Fig. 9 EVM versus driving power with no fiber and a wireless link of $2.1 \mathrm{~m}$. Constellation for $a-19.7 \mathrm{dBm}$ driven power, $b-9.2 \mathrm{dBm}$, and $c-6 \mathrm{dBm}$ 


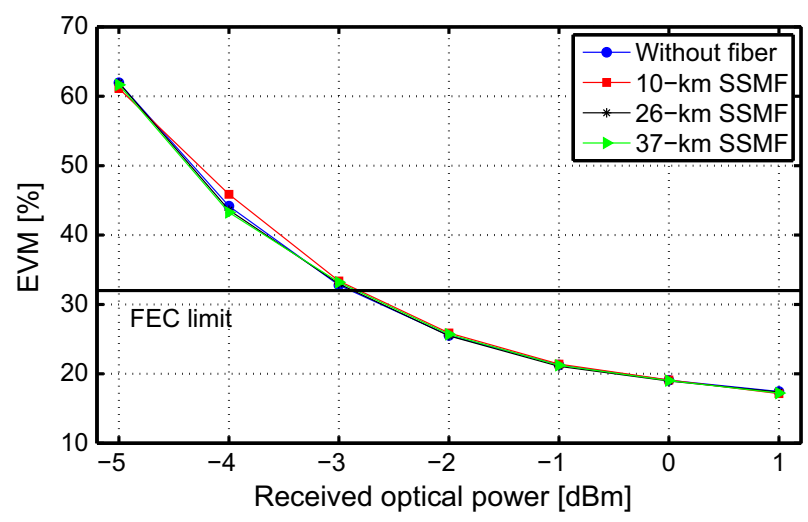

Fig. 10 Measured EVM versus received optical power for different fiber lengths and 2.1-m wireless transmission

Given the coupling between optical gain and refractive index in semiconductor lasers, it is not possible to vary the linewidth without affecting the relative intensity noise (RIN) (Buus 1990). Therefore, the impact of linewidth in the generated mm-wave was studied numerically, allowing to vary the linewidth while the RIN was kept constant. VPI Transmission Maker was used, which has semianalytical laser modules whose linewidth and RIN levels can be independently set. In these simulations no fiber was considered and the received optical power was controlled through a VOA. Figure 11 shows the EVM curves for the simulated system with different combined linewidths, together with the experimental EVM curve for a combined linewidth of $900 \mathrm{kHz}$. The match between the curves reveals that the linewidth of the laser by itself does not impact the signal EVM when an ED-based MT is employed.

\subsection{Effect of the frequency drift}

In order to assess the effect of frequency drift on the system performance, we beat two lasers whose temperature and current were set to emit at wavelengths $10-\mathrm{GHz}$ apart. In this

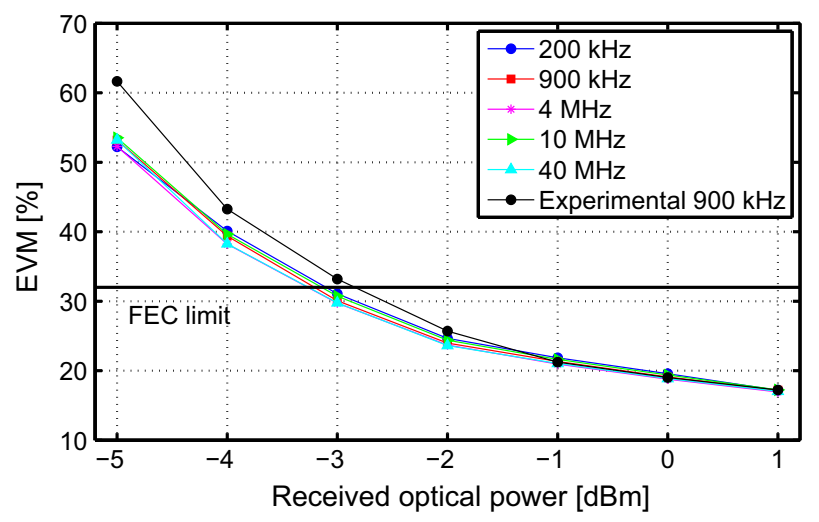

Fig. 11 Simulated EVM versus received optical power for different mm-wave carrier linewidths. The simulation results are compared to experimental measurement for $900 \mathrm{kHz}$ combined linewidth 

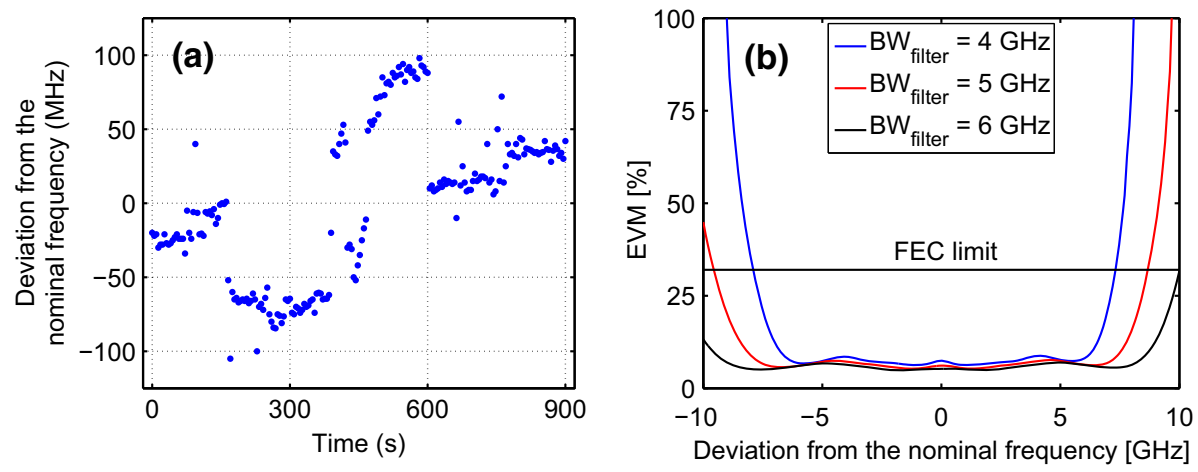

Fig. 12 a Measured long-term frequency fluctuation of a signal generated using independent lasers. b Simulated EVM in terms of the frequency deviation from the nominal frequency for different band limiting filters

way, the generated RF signal could be captured in a real-time oscilloscope. The central frequency was then measured by fitting a Lorentzian to the spectrum obtained thought the Fourier transforming the captured time series. (Aldaya et al. 2013b). The results, shown in Fig. 12a, reveal that frequency fluctuation occurs at a long time scale, i.e. tens of seconds. In addition, in the observation window, the frequency fluctuation is limited to $\pm 100 \mathrm{MHz}$, which can be attributed to the hysteresis cycle of the temperature control. On the other hand, in Fig. 12b we show the simulated EVM in terms of the frequency deviation from the nominal frequency for different band limiting filters. In this simulations, 4-th order Butterworth filters with bandwidth of 4, 5 and $6 \mathrm{GHz}$ were employed, which ensures the two sidebands to be within the transmission bandwidth. The EVM curves reveal that, as expected, the broader the filter bandwidth is, the wider the allowed detuning is. However, the shape of the EVM curves is sensibly different to the case of single carrier modulation reported in Aldaya et al. (2013a): in contrast to a sharp valley in which the EVM increases relatively fast as detuning does, in our case a broad plateau is present. This behaviour can be explained by the intrinsic robustness of OFDM signals to frequency selectivity. As a result, the system EVM remains below the FEC threshold for a detuning of $\pm 6 \mathrm{GHz}$ even for the narrower filter. Since this detuning is more than one order of magnitude higher than the measured frequency detuning, we can conclude that when ED-based MT is employed, frequency detuning results in a negligible performance degradation.

\section{Conclusion}

In this paper two different RoF systems based on photonic heterodyning techniques have been analyzed: OSBIL with a heterodyning MT and heterodyning of independent lasers with ED-based MT. Both systems have been used to generate and transmit a $640 \mathrm{Mbps}$ OFDM signal over different fiber span lengths. The system based on OSBIL has high performance in terms of power sensitivity thanks to the mixing gain of the heterodyning MT, allowing wireless link of up to $100 \mathrm{~m}$ for a fiber link of $10 \mathrm{~km}$. The combination of heterodyning technique using independent laser with ED-based MT present has poorer power sensitivity but has great potential as a cost-efficient solution for short range applications. 
Acknowledgements This work was partially supported by the São Paulo Research Foundation (FAPESP) under Grant 2015/04113-0 and the National Council of Science and Technology. Also, we want to thank Dr. Christophe Gosset for his helpful discussions and technical assistance.

\section{References}

Aldaya, I., Campuzano, G., Gosset, C., Giacoumidis, E., Doran, N., Mikroulis, S., Castañón, G.: Novel costeffective PON-to-mm-Wave RoF photonic bridge for multigigabit access networks. In: International Conference on Transparent Optical Networks (ICTON) (2013a)

Aldaya, I., Campuzano, G., Gosset, C., Castañón, G.: Phase-insensitive RF envelope detection allows optical heterodyning of MHz-linewidth signals. Photonics Technol. Lett. 25(22), 2193-2196 (2013b)

Aldaya, I., Campuzano, G., Castañón, G.: Analysis of the modulation impairments in optical sideband injection locking for millimeter-wave signal generation. J. Opt. Laser Technol. 56, 167-176 (2014a)

Aldaya, I., Campuzano, G., Gosset, C., Castañón, G.: Phase noise insensitivity of RF envelope detection and its application to OFDM radio over fiber systems. J. Opt. Laser Technol. 58, 139-144 (2014b)

Andrews, J.G., Ghosh, A., Muhamed, R.: Fundamentals of WiMAX. Prentice Hall, Englewood Cliffs (2007)

Beas, J., Castañón, G., Aldaya, I., Campuzano, G., Aragón-Zavala, A.: Millimeter-wave frequency radio over fiber: a survey. IEEE Commun. Surv. Tutor. 99, 1-27 (2013)

Bedrosian, E.: Memorandum RM-3439-PR A product theorem for Hilbert transform. Technical Report, United States Air Force (project RAND) (1962)

Berceli, T., Herczfeld, P.R.: Microwave photonics-a historical perspective. IEEE Trans. Microw. Theory Tech. 58(11), 2992-3000 (2010)

Bhattacharya, M., Sarkar, B., Chattopadhyay, T.: Optical generation of millimeter and submillimeter-waves through optical side-band injection locking of semiconductor lasers. IEEE Photonics Technol. Lett. 4(11), 1611-1613 (2002)

Braun, R.P.: Tutorial: fibre radio systems, applications and devices. In: Proceedings of 24th European Conference on Optical Communication (ECOC) (1998)

Brillant, A.: Digital and Analog Fiber Optic Communications for CATV and FTTx Applications. SPIE Publishing Group, Bellingham (2008)

Buus, J.: Single Frequency Semiconductor Lasers. SPIE Optical Engineering Press, Bellingham (1990)

Daniels, R.C., Robert, W., Heath, J.: $60 \mathrm{GHz}$ wireless communications: emerging requirements and design recommendations. IEEE Veh. Technol. Mag. 2, 41-50 (2007)

Gallion, P., Debarge, G.: Quantum phase noise and field correlation in single frequency semiconductor laser systems. IEEE J. Quantum Electron. 20(4), 343-349 (1984)

Garcia-Armada, A.: Understanding the effects of phase noise in orthogonal frequency division multiplexing (OFDM). IEEE Trans. Broadcast. 47(2), 153-159 (2001)

Ghafouri-Shiraz, H.: Distributed Feedback Laser Diodes and Optical Tunable Filters. Wiley, London (2003)

Giannetti, F., Luise, M., Reggiannini, R.: Mobile and personal communications in the $60 \mathrm{GHz}$ band: a survey. Wirel. Pers. Commun. 10(2), 207-243 (1999)

Gliese, U., Norskov, S., Nielsen, T.: Chromatic dispersion in fiber-optic microwave and millimeter-wave links. IEEE Trans. Microw. Theory Tech. 44(10), 1716-1724 (1996)

Goldberg, L., Taylor, H., Weller, J., Bloom, D.: Microwave signal generation with injection-locked laser diodes. IEE Electron. Lett. 19(13), 491-493 (1983)

González-Insua, I., Schaffer, C.G., Christian G.: Heterodyne radio over fiber system with 10 Gbps data rates. In: Optical Fiber Communication Conference (OSA) (2009)

González-Insua, I., Plettemeier, D., Schaffer, C.G.: Simple remote heterodyne radio over fiber system for Gbps wireless access. J. Lightw. Technol. 28(13), 2289-2295 (2010)

Hall, T.J., Maldonado-Basilio, R., Abdul-Majid, S., Seregelyi, J., Li, R., Antolín-Pérez, I., Nikkhah, H., Lucarz, F., de Bougrenet de La Tocnaye, J.L., Fracasso, B., Pajusco, P., Karnfelt, C., Bourreau, D., Ney, M., Guemri, R., Josse, Y., Liu, H.: Radio-over-fibre for green wireless access networks. Ann. Telecommun. 68, 3-21 (2013)

Hansen, C.: WiGiG: multi-gigabit wireless communications in the $60 \mathrm{GHz}$ band. IEEE Wirel. Commun. 18(6), 6-7 (2011)

Hwang, T., Yang, C., Wu, G., Li, S., Ye Li, G.: OFDM and its wireless applications: a survey. IEEE Trans. Veh. Technol. 58(4), 1673-1694 (2009)

Jia, Z., Yu, J., Qian, D., Ellinas, G., Chang, G.K.: Experimental demonstration for delivering 1-Gb/s OFDM signals over $80-\mathrm{km}$ SSMF in 40-GHz radio-over-fiber access systems. In: Proceedings of the Conference on Optical Fiber Communication (OFC), pp. 1-3 (2008) 
Khawaja, B.A., Cryan, M.J.: Wireless hybrid mode locked lasers for next generation radio-over-fiber systems. J. Lightw. Technol. 28(16), 2268-2276 (2010)

Liu, Y., Davies, A., Ingham, J., Penty, R., White, I.: Nanometer scale thermal drift of 4Gbit/s uncooled laser for tight-channel-spacing low-cost WDM. In: Conference on Laser and Electro-optics (OSA) (2005)

Logan, R.T.: Fiber-optic delay-line stabilization of heterodyne optical signal generator and method using same. US5687261 United States Patent (1997)

Lowery, A.J.: Transmission-line modelling of semiconductor lasers: the transmission-line laser model. Int. J. Numer. Model. Electron. Netw. Devices Fields 2, 249-265 (1989)

Marcu, C., et al.: A $90 \mathrm{~nm}$ CMOS low-power $60 \mathrm{GHz}$ transceiver with integrated baseband circuitry. IEEE J. Solid State Circuits 44(12), 3434-3447 (2009)

Ng'oma, A., Sauer, M.: Radio-over-fiber systems for multi-Gbps wireless communication. In: Proceedings of the Communications and Photonics Conference and Exhibition (ACP), pp. 1-2 (2009)

Okajima, Y., Sheng-Kuang, H., Liu, J.: Experimental observation of chirp reduction in bandwidht-enhanced semiconductor lasers subject to strong optical injection. Opt. Commun. 219(1-6), 357-364 (2003)

Part 15.3: Wireless medium access control (MAC) and physical layer (PHY) specifications for high rate wireless personal area networks (WPANs). Amendment 2: Millimeter-wave-based Alternative Physical Layer Extension. (2009)

Rappaport, T.: Millimeter wave cellular: a road to 5G. In: IEEE International Conference on Communications (ICC), p. 317 (2013)

Rappaport, T.: Antennas and Propagation for Wireless Communication Systems, 2nd edn. Wiley, London (2007)

Saunders, S., Aragon-Zavala, A.: Antennas and Propagation for Wireless Communication Systems. Wiley, London (2007)

Shafik, R., Rahman, M.S., Islam, A.R.: On the extended relationships among EVM, BER and SNR as performance metrics. In: Proceeding of International Conference on Electrical and Computer Engineering (ICE) (2006)

Shieh, W., Djordjevic, I.: OFDM for Optical Communications. Academic Press, Elsevier, London, Amsterdam (2010)

Standard ECMA-387: High rate $60 \mathrm{GHz}$ PHY, MAC, and PALs (2010). https://www.ecma-international. org/publications/files/ECMA-ST/ECMA-387.pdf

Timofeev, F., Bennett, S., Griffin, R., Bayvel, P., Seeds, A., Wyatt, R., Kashyap, R., Robertson, M.: High spectral purity millimetre-wave modulated optical signal generation using fibre grating lasers. In: Digest of IEEE International Microwave Symposium (MTT-S), vol. 3, pp. 1221-1224 (1998)

Wake, D., Lima, C., Davies, P.: Optical generation of millimeter-wave signals for fiber-radio systems using a dual-mode DFB semiconductor laser. IEEE Trans. Microw. Theory Tech. 43(9), 2270-2276 (1995)

Zou, Q., Tarighat, A., Sayed, A.: Compensation of phase noise in OFDM wireless systems. IEEE Trans. Signal. Process. 55(11), 5407-5424 (2007) 